Trauma Berufskrankh 2014 • 16[Suppl 4]:425-430 DOI 10.1007/s10039-014-2101-7

Online publiziert: 17. September 2014

c) Springer-Verlag Berlin Heidelberg 2014

B. Bohn ${ }^{1,2}$ - A.S. Gonschorek ${ }^{3}$ G. Kammler ${ }^{4}$ C. Jürgens ${ }^{1}$

${ }^{1}$ Abteilung für Unfallchirurgie, Orthopädie und Sporttraumatologie, BG-Unfallkrankenhaus Hamburg

2 Katholisches Kinderkrankenhaus Wilhelmstift, Hamburg

${ }^{3}$ Neurotraumatologisches Zentrum, BG-Unfallkrankenhaus Hamburg

${ }^{4}$ Klinik für Neurochirurgie, Universitätskrankenhaus Hamburg-Eppendorf

\title{
Management des Schädel-Hirn-Traumas bei Kindern und Jugendlichen
}

fige Ursachen eines SHT im Kindesalter, und auch ein scheinbares Bagatelltrauma kann bei dieser Altersgruppe ein schweres SHT verursachen.

Kinder haben eine Neigung zu ausgeprägten Hirnschwellungen.

Gerinnungsstörungen, auch infolge einer Dauermedikation, sowie begleitende Intoxikationen als Ursache einer Bewusstseinstrübung sind differenzialdiagnostisch bei Kindern seltener als bei Erwachsenen zu berücksichtigen.

\section{Konkussion (sog.}

\section{Gehirnerschütterung) beim Sport}

Das Schicksal des 13-jährigen Footballspielers Zachery Lystedt, der 2008 durch ein repetitives SHT Grad 1 ein „second impact syndrom" erlitten hatte, schärfte das Bewusstsein für Präventionsmaßnahmen beim Sport zunächst in den USA, in der Folge auch weltweit.

Das Zachery-Lystedt-Law kommt heute in den USA nahezu flächendeckend zur Anwendung. Kurz zusammengefasst besagt es, dass Athleten, Eltern und Trainer jährlich über die Gefahren einer Hirnverletzung beim Sport unterrichtet werden müssen. Ein Kind oder Jugendlicher mit einem leichten SHT muss aus dem Spiel genommen werden.

Teamsportarten wohnt ein höheres Risiko für ein SHT inne. Die SCAT-Card
(SCAT: „sport concussion assessment tool") kommt schon heute bei vielen professionellen Sportveranstaltungen zur Anwendung. Eine Anpassung der medizinischen Diagnostik, aber auch der Gesetzgebung, beim leichten SHT im Jugendsport ist auch in Europa zu erwarten.

\section{Klassifikation}

Eine Schädelprellung ist Folge einer Gewalteinwirkung auf den Schädel ohne Fraktur, Bewusstlosigkeit oder neurologische Symptome/Hirnfunktionsstörungen.

Bei der Klassifikation des SHT wird international am häufigsten die Einteilung in die 3 Schweregrade

- leicht/Grad I,

- mittelschwer/Grad II und

- schwer/Grad III

benutzt, die sich auf die in der GCS (Glasgow-Coma-Scale) erfassten neurologischen Teilbefunde und den resultierenden Summenscore bezieht [24]. Bei der GCS werden verbale Antwort, motorische Antwort und das Öffnen der Augen geprüft. Dabei werden bei der Beurteilung von Kindern in einem Alter unter 24 Monaten statt der verbalen Antwort, die entwicklungsbedingt eingeschränkt ist, Mimik, Erweckbarkeit, Trinkverhalten sowie Spontanmotorik bewertet. Bei Kindern ab dem 24. Lebensmonat wird die den Kopf einwirkenden Gewalteinflüssen. Stürze und Sportunfälle sind daher häu- 


\section{Besonderheiten der Kindertraumatologie}
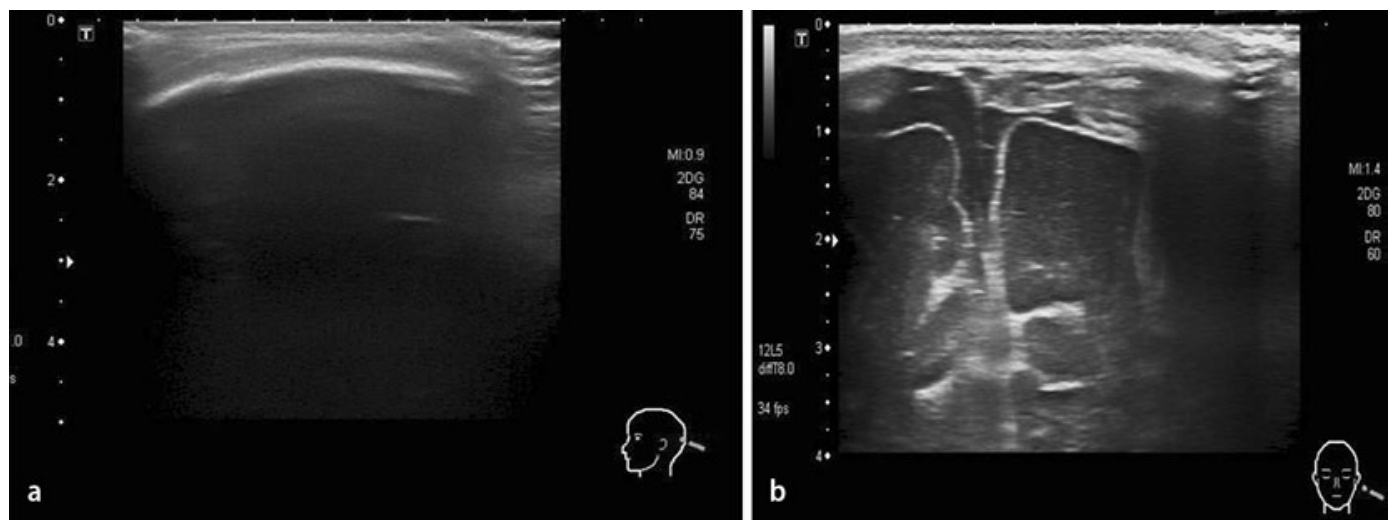

Abb. $1<$ Sonografie des Schädels bei 4 Monate altem Säugling, nach „Sturz auf dem Arm der Mutter", a Kalottenfraktur parietal, b subdurales Hämatom bis $8 \mathrm{~mm}$ Tiefenausdehnung, GCS 13 (fixiert inkonstant, gezielte Abwehr auf Schmerzreize, Augenöffnen spontan), GCS Glasgow-Coma-Scale
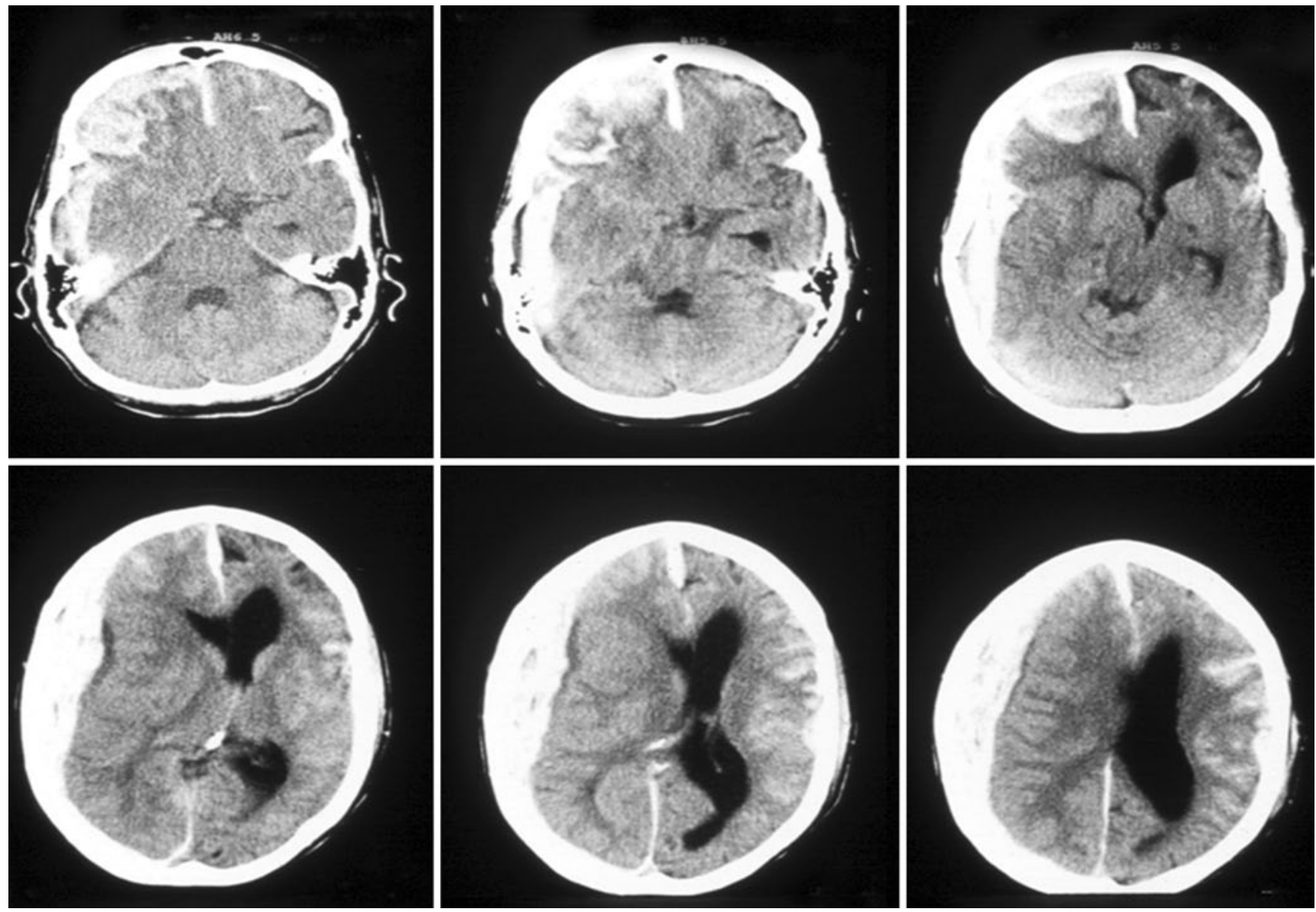

Abb. $2 \Delta$ CT des Schädels bei 14-jährigem, als Fußgänger vom PKW erfasstem Jungen: akutes subdurales Hämatom rechts mit typischer Konfiguration und Mittellinienverlagerung

verbale Interaktionsfähigkeit wie bei Jugendlichen und Erwachsenen eingestuft. Die maximal erreichbare Punktzahl bei der verbalen Antwort beträgt 5 Punkte. Bei der motorischen Antwort wird für die Erreichbarkeit der maximalen Punktzahl von 6 bei Kindern unter 24 Monaten gefordert, dass sie gezielt nach Gegenständen greifen, bei älteren Kindern wie bei Erwachsenen, dass sie Aufforderun- gen befolgen. Die Beurteilung der Augenöffnung unterscheidet sich bei jungen und älteren Kindern sowie Erwachsenen nicht, dabei sind maximal 4 Punkte erreichbar.

Die GCS hat methodische Schwächen, so ist die Summenbildung nicht immer möglich. Auch die Abhängigkeit vom Ausbildungsstand der Untersucher und die Beeinflussung durch die angewandte
Therapie stehen in der Kritik. Die Anwendung der GCS für Therapieentscheidungen und Prognosestellungen bedarf insbesondere bei Kindern einer Modifikation $[8,10,17,21,25]$.

Ein leichtes SHT liegt bei einem GCSWert von 13 bis 15 Punkten, das mittelschwere SHT bei 9 bis 12 Punkten und das schwere SHT bei weniger als 9 Punkten vor. 
Die früher verwendete Einstufung des SHT nach der Dauer der Bewusstlosigkeit hat aufgrund der oft unsicheren Angaben keine wesentliche Bedeutung mehr.

Unterschieden werden außerdem geschlossenes und offenes SHT. Bei Letzterem liegt neben der Weichteilschädigung und der Schädelfraktur auch eine Duraverletzung vor.

\section{Präklinische Diagnostik und präklinisches Management}

Der GCS-Wert sollte aufgrund der möglichen dynamischen Entwicklung des SHT idealerweise am Unfallort, nach Stabilisierung, beim Eintreffen im Krankenhaus und in anfänglich engmaschigen Verlaufskontrollen erhoben und dokumentiert werden.

Neben der Beurteilung nach GCS sollten Wesensveränderungen wie Schläfrigkeit, Spielunlust, Inappetenz und verlangsamte Reaktionen beachtet werden, wobei sich diese Symptome vom Untersucher gelegentlich nur schwer erfassen lassen [8]. Auch erfragte Amnesie, auffällige Pupillenreaktionen und andere vegetative Symptome wie Kopfschmerzen, Schwindel, Übelkeit, Erbrechen und Sehstörungen sollten für die Beurteilung des dynamischen Verlaufs protokolliert werden. Dabei kommt gerade bei Kleinkindern den Angaben der Bezugspersonen eine große Bedeutung zu [14]. Nach Verletzungszeichen wie Schwellung, Hämatomen, Wunden, Skalpierung, Schädeldeformitäten, Austritt von Liquor oder Hirngewebe und Blutungen aus Ohr, Nase und Mund sollte gezielt gesucht werden.

Die Klinikeinweisung bzw. ein Transport in die Klinik sollten erfolgen bei:

- Koma,

- Bewusstseinstrübung,

- neurologischen Ausfällen,

- Krampfanfall,

- Hinweis auf Schädelfraktur und

- Liquoraustritt aus Ohr/Nase.

Die Einweisung ist ratsam bei:

- Erbrechen,

- starken andauernden Kopfschmerzen,

- Verdacht auf Kindesmisshandlung (sog. Schütteltrauma) und
- Verhaltensänderungen bei Kindern unter 24 Lebensmonaten.

Am Unfallort erfolgt die Sicherung der Vitalfunktionen mit dem Ziel der Normovolämie und Normotonie. Bei Infusionsbedürftigkeit sollte mit isotoner $\mathrm{NaCl}$ Infusionslösung ein mittlerer arterieller Druck $>12 \mathrm{kPa}(>90 \mathrm{mmHg})$ angestrebt werden [8].

Bei GCS $<9$ oder respiratorischer Gefährdung, z. B. durch Mittelgesichtsfrakturen, sollten gerade Kinder intubiert werden, da sie schnell dekompensieren können. Auch andere atmungslevante Begleitverletzungen wie Pneumothorax und Hämatothorax müssen erkannt und entsprechend behandelt werden.

Bei der Beatmung ist eine unkontrollierte Hyperventilation zu vermeiden. Ein Absinken der arteriellen Sauerstoffsättigung unter $90 \%$ sollte vermieden werden [11].

Bei einem GCS-Wert $<9$ sollte die Zielklinik eine Kinderklinik/Klinik mit Zulassung zum Schwerstverletzungsartenverfahren (SAV) mit Computertomograf (CT) und neurochirurgischer Abteilung mit 24-stündiger Verfügbarkeit sein.

\section{Diagnostik und Therapie in der Klinik}

Ziel der Therapie ist es, den sekundären Hirnschaden durch Blutung, Ischämie, Ödem, entzündliche Veränderungen, gestörte zerebrale Autoregulation mit Hirndruckanstieg, Ischämie und neuronalem Zelltod zu verhindern oder zumindest zu minimieren.

Nach der Akutversorgung am Unfallort und während des Transports und der weiteren Diagnostik in der Klinik sind folgende Maßnahmen indiziert:

\section{Nicht bewusstloses Kind mit leichtem SHT}

- Überwachung mit Monitoring über 12-48 h,

- ggf. Infusion,

- ggf. Sonografie des Schädels,

- ggf. CT/MRT (Magnetresonanztomografie) des Schädels [8, 14, 18].
Trauma Berufskrankh 2014 - 16[Suppl 4]:425-430

DOI 10.1007/s10039-014-2101-7

C) Springer-Verlag Berlin Heidelberg 2014

B. Bohn · A.S. Gonschorek · G. Kammler C. Jürgens

Management des SchädelHirn-Traumas bei Kindern und Jugendlichen

\section{Zusammenfassung}

Hintergrund. Das Schädel-Hirn-Trauma (SHT) ist trotz erfolgreicher Präventionsmaßnahmen weiterhin die häufigste traumatische Ursache für Morbidität und Mortalität bei Kindern und Jugendlichen. Bei Kindern können auch scheinbare Bagatelltraumen ein schweres SHT verursachen.

Diagnostik und Versorgung. Die dynamische neurologische Symptomatik ist zu beachten und engmaschig zu kontrollieren. Auch beim leichten SHT sollte bei Zweifeln an der Prognose eine Klinikeinweisung mit kindgerechter Überwachungsmöglichkeit, adäquater bildgebender Diagnostik und Therapie erfolgen. Beim schweren SHT ist eine Zielklinik mit neurochirurgischer Abteilung und 24-stündiger Verfügbarkeit auszuwählen. Das schwere SHT bei Kindern ist selten, die Prognose trotz Therapie aufgrund der primären Hirnschädigung immer noch ungünstig.

\section{Schlüsselwörter}

Kinder · Kopfverletzung $\cdot$ Hirnverletzungen · Intrakranialer Druck - Glasgow-Coma-Scale

\section{Management of head trauma in children}

\section{Abstract}

Background. Despite successful prevention measures, head trauma in children is still the most common traumatic cause of morbidity in children and adolescents. In children, seemingly trivial traumas can also cause severe brain injury.

Diagnosis and treatment. The dynamic neurological symptoms need to observed and closely monitored. Even in light head injury, hospitalization with pediatric monitoring capabilities, adequate diagnostic imaging, and therapy is recommended if there is doubt about the prognosis. In cases with severe brain injury, a clinic with a neurosurgical department with 24-h availability is to be selected. Although severe brain injuries in children are rare, the prognosis is still unfavorable despite therapy because of primary brain damage.

\section{Keywords}

Children · Head injury · Brain injuries .

Intracranial pressure $\cdot$ Glasgow coma scale 


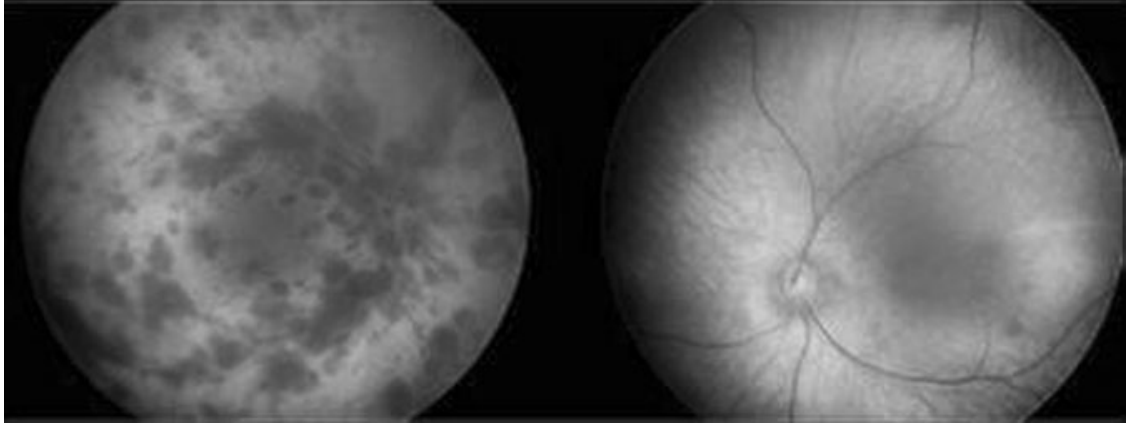

Abb. $3 \Delta$ Fundoskopie bei Kind mit Schütteltrauma und Hämorrhagien rechts

Die konservative Therapie beim SHT Grad I beinhaltet Bettruhe unter Monitoring über 12-48 $\mathrm{h}$ mit anfänglich 1- bis 2-stündlicher Kontrolle mit Dokumentation auf einem entsprechenden Protokoll. Bei Übelkeit und/oder Erbrechen sollte ggf. isotone $\mathrm{NaCl}$-Lösung infundiert werden.

Nach der Entlassung aus der stationären Behandlung empfehlen wir Schonung über 3 Tage, Einschränkung des Fernsehkonsums, der Nutzung von Computerspielen und Sportkarenz bis zum kompletten Rückgang der Symptome (sog. gute Praxis).

Ist die verantwortliche betreuende Person zuverlässig und eine rasche Wiedervorstellung möglich, kann abhängig von der Art des Traumas und bei unauffälligem neurologischem Verlauf auch überlegt werden, ob im Einzelfall von einer stationären Aufnahme nach entsprechend ausführlicher mündlicher und schriftlicher Aufklärung abgesehen werden kann.

\section{Bewusstloses Kind nach Stabilisierung der Vitalparameter}

- Schockraummanagement (ATLS: „advanced trauma life support“):

- FAST („focused assessment with sonography for trauma“),

- (Ganzkörper)-CT,

- Labor mit Blutbild, Gerinnungsstatus, Elektrolyten, Glukose, Blutgruppe, Leber-, Nieren- und Pankreaswerten

Röntgenaufnahmen des Schädels in 2 Ebenen erfolgen nur in Ausnahmefällen, ggf. als Spezialaufnahmen, da sie keine ausreichenden Informationen für die weitere Therapie liefern. Intrakraniale
Verletzungen können durch sie nicht ausgeschlossen werden [16], und ein Frakturnachweis in konventionellen Röntgenaufnahmen ändert häufig nichts am Vorgehen im Verlauf.

Die transfontanellare oder transkraniale Sonografie (• Abb. 1) des Schädels hingegen bietet zusätzliche Informationen, falls eine CT nicht zwingend notwendig ist $[15,22]$.

Ein kraniales CT sollte durchgeführt werden bei:

- Koma,
- GCS $<14$,
- fokalen neurologischen Defiziten,
- Verdacht auf Kalottenfraktur und
- neurologischer Verschlechterung.

Die MRT stellt in der Akutdiagnostik gegenüber der CT ( $\bullet$ Abb. 2) einen zu hohen apparativen und zeitlichen Aufwand dar, bietet in der Verlaufsdiagnostik des SHT aber Vorteile bei der Beurteilung parenchymatöser Veränderungen wie Ödem, diffus axonales Trauma, Kontusionen, Ischämien und Blutungen.

Bei der CT werden Frakturen und subarachnoidale Blutungen im Vergleich zur MRT besser detektiert. Die hohe Strahlenbelastung durch die CT sollte bei der Indikationsstellung jedoch berücksichtigt werden.

\section{Schütteltrauma}

Bei Kindern vor dem 3. Lebensjahr mit unklarer Anamnese und einem Missverhältnis zum neurologischen Status muss an ein Schütteltrauma gedacht werden. Bezüglich der Inzidenz besteht eine hohe Dunkelziffer. Auch die Rate der neurologischen Folgeschäden ist hoch.
Bei der Spiegelung des Augenhinter-

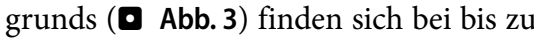
$21 \%$ der Opfer asymmetrische/einseitige Hämorrhagien.

Eine Elektroenzephalografie (EEG) kann Allgemeinstörungen oder Herdbefunde als Folge einer funktionellen oder strukturellen Hirnschädigung aufweisen und prognostische Hinweise geben, stellt aber kein Akutdiagnostikum außer bei der Hirntoddiagnostik bei schwerstem SHT dar [4].

\section{Therapie beim SHT Grad III}

\section{Konservative Behandlung}

Das Hirndruckmanagement zur Vermeidung sekundärer Hirnschäden durch Senkung des intrakranialen Drucks (ICP) erfolgt unter Beatmung auf der Intensivstation. Voraussetzung einer ausreichenden Hirndurchblutung ist ein entsprechender zerebraler Perfusionsdruck (CPP), der sich aus der Differenz des mittleren arteriellen Blutdrucks und des mittleren ICP errechnet. Die derzeit vorliegende Evidenz spricht dafür, dass der CPP im Kindesalter nicht unter 5,33 $\mathrm{kPa}(40 \mathrm{mmHg})$ sinken sollte. Bei einem Zielwert von $8 \mathrm{kPa}(60 \mathrm{mmHg}$ ) sollte er aber nicht über $9,33 \mathrm{kPa}(70 \mathrm{mmHg})$ angehoben werden $[1,5,6]$.

Ziel der konservativen Therapie, basierend auf einer allenfalls leichten Hyperventilation mit einem $\mathrm{CO}_{2}$-Zielwert von $4,67 \mathrm{kPa}(35 \mathrm{mmHg})$, Lagerung mit etwa $30^{\circ}$ Oberkörperhochlagerung und Analgosedierung ist das Erreichen einer Homöostase. Ausgefallene Funktionen wie Atmung, Nahrungsaufnahme und Flüssigkeitszufuhr müssen entsprechend übernommen werden.

Von fraglichem hirnprotektivem Nutzen sind hypertone NaCl-Infusionslösungen [25], Barbituratnarkose und Hypothermie $[3,13,25]$. Osmodiuretika wie Mannitol können eine kurzzeitige Senkung des ICP bewirken, dabei müssen aber die Serumosmolarität und die Nierenfunktion überwacht werden $[5,19]$. Auf die Medikation mit Glukokortikoiden sollte wegen der erhöhten Letalität aufgrund von Infektionen und gastrointestinalen Blutungen verzichtet werden [2, 9]. 

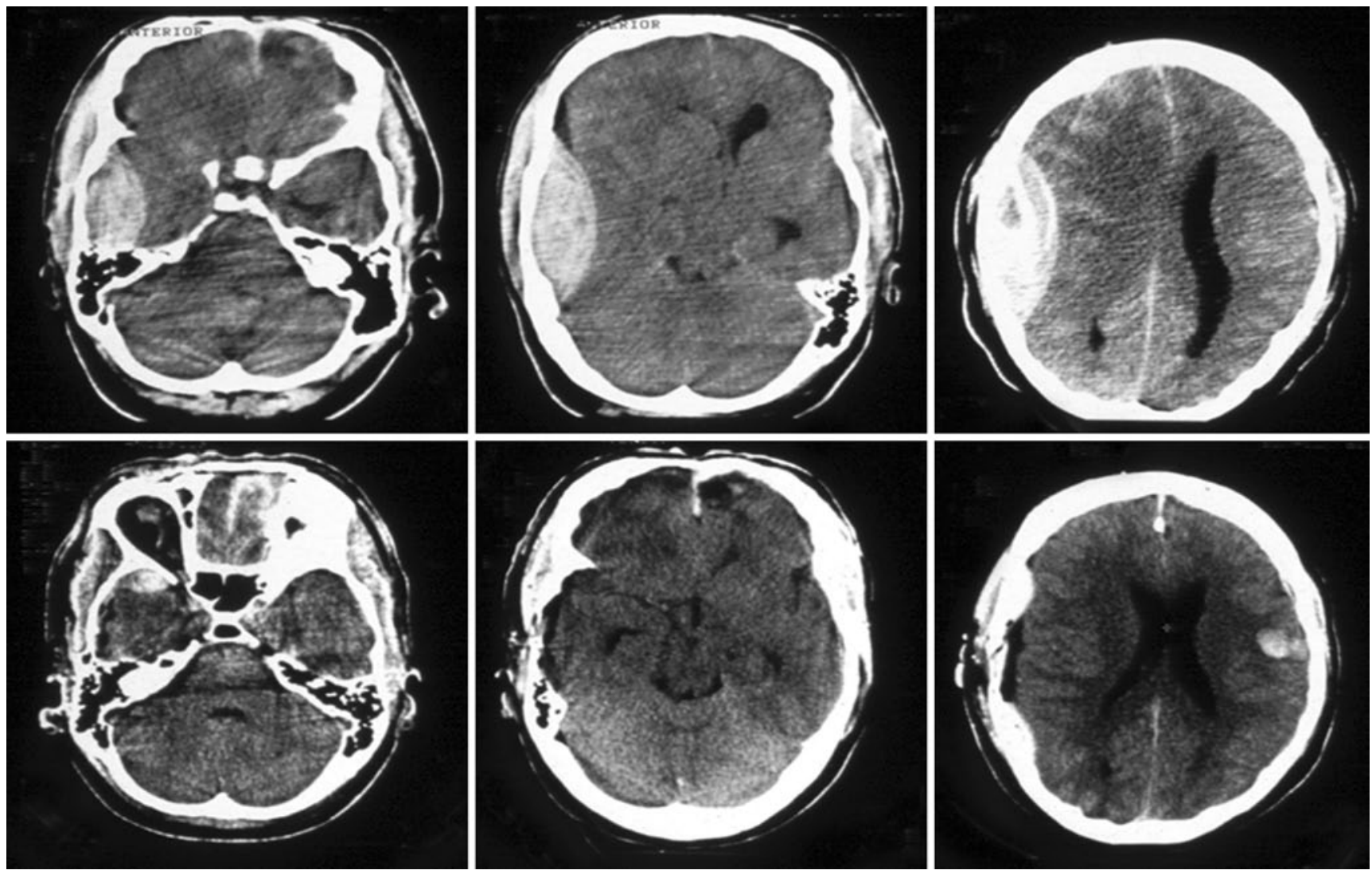

Abb. $4 \Delta$ Epidurales Hämatom temporal rechts mit typischer bikonvexer Konfiguration vor (obere Reihe) und nach (untere Reihe) neurochirurgischer Entlastung bei 12-jährigem Mädchen, Sturz mit Inlineskates
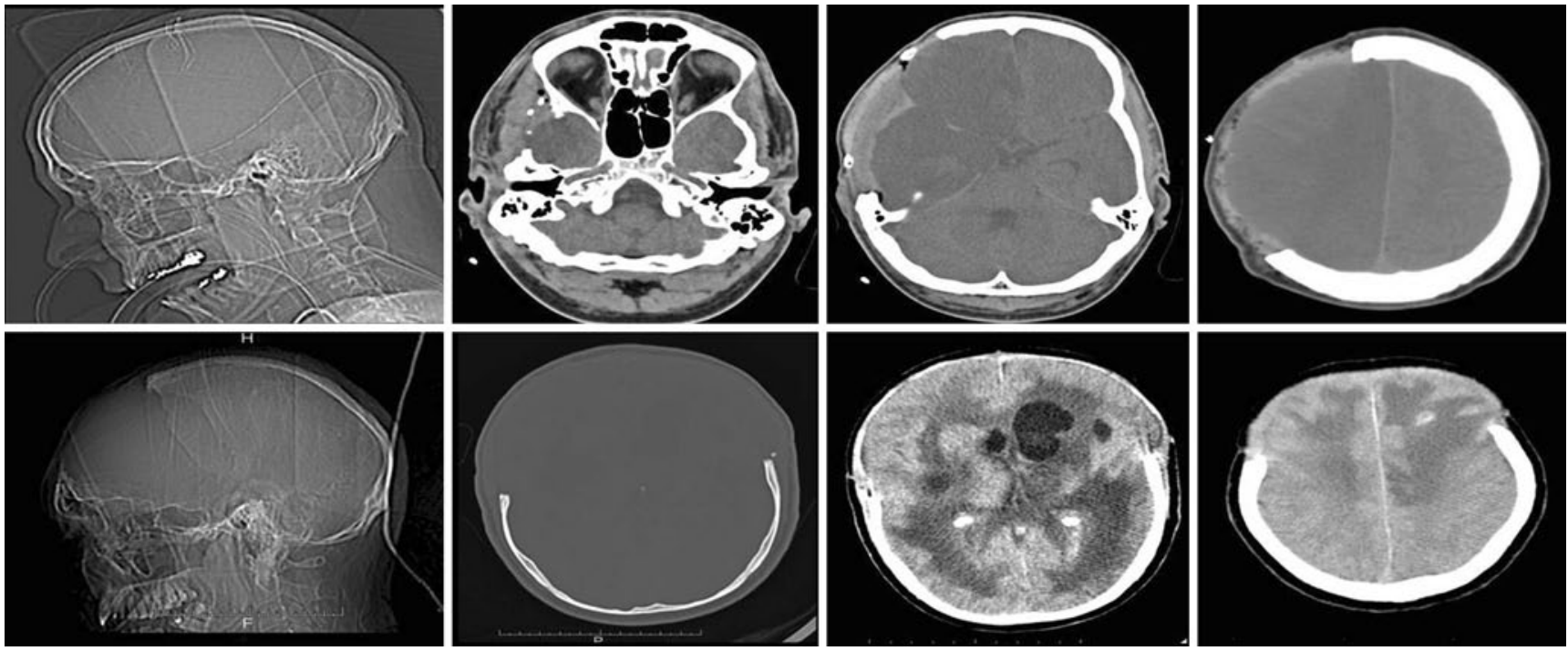

Abb. $5 \Delta$ Dekompressive Kraniektomie

\section{Operative Behandlung}

Die Indikationsstellung für die Dringlichkeit und Technik der operativen Entlastung einer traumatischen intrakranialen Raumforderung mit Verlagerung zerebraler Strukturen, insbesondere des normalerweise in der Mittellinie gelegenen
3. Ventrikels (- Abb. 4), sollte neben dem Befund in der CT auch die Dynamik der klinischen Symptomatik berücksichtigen.

Der Zeitpunkt der Operation wird vom neurochirurgischen Behandlungsteam, individuell entsprechend der Situation des Patienten, festgelegt.
Eine wirksame Möglichkeit, den erhöhten intrakranialen Druck zu senken, ist die operative Dekompression durch Kraniektomie und ggf. Duraerweiterungsplastik (• Abb. 5).

Einzelne Studien zeigten gute Behandlungsergebnisse [20], für eine abschlie- 
ßende Bewertung sind jedoch weitere wissenschaftliche Untersuchungen notwendig.

Die Prognose bei entsprechend schwerem SHT ist trotz aller intensivmedizinischen, neurochirurgischen und anschließenden rehabilitativen Behandlungen ungünstig. Dies sollte Motivation sein, auch weiterhin prophylaktische Maßnahmen zur Verhinderung eines SHT zu unterstützen.

\section{Fazit für die Praxis}

- Das leichte SHT bei Kindern und Jugendlichen ist häufig.

- Auch beim leichten SHT sollte wegen der möglichen erheblichen Folgen und der dynamischen neurologischen Entwicklung die Vorstellung in einer geeigneten Klinik erfolgen.

- Beim schweren SHT muss eine neurochirurgische Behandlungsmöglichkeit jederzeit gewährleistet sein.

- An mögliche Kindesmisshandlung als Ursache eines SHT ist zu denken.

- Der primäre Hirnschaden ist irreversibel, funktionsgestörte Neuronen können aber regenerieren.

- Durch eine adäquate Therapie kann die Entwicklung sekundärer Hirnschäden begrenzt werden.

\section{Korrespondenzadresse}

\section{Dr. B. Bohn}

Abteilung für Unfallchirurgie, Orthopädie und Sporttraumatologie, BG-Unfallkrankenhaus Hamburg, Bergedorfer Straße 10, 21033 Hamburg b.bohn@kkh-wilhelmstift.de

\section{Einhaltung ethischer Richtlinien}

Interessenkonflikt. B. Bohn, A.S. Gonschorek, G. Kammler und $C$. Jürgens geben an, dass kein Interessenkonflikt besteht.

Dieser Beitrag beinhaltet keine Studien an Menschen oderTieren.

The supplement containing this article is not sponsored by industry.

\section{Literatur}

1. Adelson PD, Bratton SL, Carney NA et al (2003) Guidelines for the acute medical management of severe traumatic brain injury in infants, children and adolescents. Chapter 11. Use of hyperosmolar therapy in the management of severe pediatric brain injury. Pediatr Crit Care Med 4/3:40-44

2. Alderson P, Roberts I (2008) Corticosteroids for acute traumatic brain injury. Cochrane Database Syst Rev 1:CD000196

3. AldersonXX P, Gadkary C, Signorini DF (2004) Therapeutic hypothermia for head injury. Cochrane Database Syst Rev 4:CD001048.pub2. DOI 10.1002/14651858. CD001048.pub2

4. Bagnato S, Boccagni C, Prestandrea C et al (2010) Prognostic value of standard EEG in traumatic and non-traumatic disorders of consciousness following coma. Clin Neurophysiol 121:274-280

5. Brain Trauma Foundation, The American Association of Neurological Surgeons, The Joint Section on Neurotrauma and Critical Care (2007) Guidelines for the management of severe traumatic brain injury. J Neurotrauma [Suppl 1] 24:91-95

6. Catalana-Temprano A, Claret Teruel G, Cambra Lasaosa FJ et al (2007) Intracranial pressure and cerebral perfusion pressure as risk-factors in children with traumatic brain injuries. Neurosurgery 106:463-466

7. Deutsche Gesellschaft für Neurologie (2008) Leichtes Schädel-Hirn-Trauma. AWMF-Leitlinienregisternummer 030/047. AWMF, Düsseldorf

8. Dunning J, Daly JP, Lomas JP et al; Children's Head Injury Algorithm for the Prediction of Important Clinical Events Study Group (2006) Derivation of the children's head injury algorithm for the prediction of important clinical events decision rule for head injury in children. Arch Dis Child 91:885-891

9. Edwards $P$, Arango M, Balica L et al (2005) Final results of MRC CRASH, a randomised placebo controlled trial of intravenous corticosteroids in adults with head injury - outcomes at 6 months. Lancet 365:1957-1959

10. Fortune PM, Shann F (2010) The motor response to stimulation predicts outcome as well as the full Glasgow Coma Scale in children with severe head injury. Pediatr Crit Care Med 11:339-342

11. Gabriel EJ, Ghajar J, Jagoda A et al (2002) Brain trauma foundation guidelines for prehospital management of traumatic brain injury. J Neurotrauma 19:111-114

12. Gesellschaft für Neonatologie und Pädiatrische Intensivmedizin, Deutsche Gesellschaft für Kinderchirurgie, Gesellschaft für Neuropädiatrie, Deutsche Gesellschaft für Neurochirurgie, Deutsche Gesellschaft für Neuroradiologie, Gesellschaft für Pädiatrische Radiologie, Deutsche Gesellschaft für Anästhesie und Intensivmedizin, Deutsche Gesellschaft für Unfallchirurgie, Deutsche Interdisziplinäre Vereinigung für Intensiv- und Notfallmedizin (2011) Das Schädel-Hirn-Trauma im Kindesalter. AWMF-Leitlinienregister Nr. 024/018. AWMF, Düsseldorf

13. Harris OA, Colford JM Jr, Good MC, Matz PG (2002) The role of hypothermia in the management of severe brain injury: a meta-analysis. Arch Neurol 59:1077-1083

14. Kuppermann N, Holmes JF et al (2009) Identification of children at very low risk of clinically important brain injuries after head trauma: a prospective cohort study. Lancet 374:1160-1170
15. Mandera M, Larysz D, Wojtacha M (2002) Changes in cerebral hemodynamics assessed by transcranial Doppler ultrasonography in children after head injury. Childs Nerv Syst 18:124-128

16. Masters SJ, McClean PM, Arcarese JS et al (1987) Skull X-ray examinations after head trauma. Recommendations by a multidisciplinary panel and validation study. N Engl J Med 316:84-91

17. Ong L, Selladurai BM, Dhillon MK et al (1996) The prognostic value of the Glasgow Coma Scale, hypoxia and computerised tomography in outcome prediction of pediatric head injury. Pediatr Neurosurg 24:285-291

18. Osmond MH, Klassen TP, Wells GA et al (2010) Catch: a clinical decision rule for the use of computed tomography in children with minor head injury. CMAJ 182:341-348

19. Roberts I, Schierhout G, Wakai A (2003) Mannitol for acute traumatic brain injury. Cochrane Database Syst Rev 2:CD001049

20. Sahuquillo J, Arikan F (2006) Decompressive craniectomy for the treatment of refractory high intracranial pressure in traumatic brain injury. Cochrane Database Syst Rev 1:CD003983.pup2. DOI 10.1002/14651858. CD003983.pub2

21. Schnadower D, Vazquez H, Lee J et al (2007) Controversies in the evaluation and management of minor blunt head trauma in children. Curr Opin Pediatr 9:258-264

22. Sert A (2007) Cranial ultrasound as a non-invasive diagnostic technique in the diagnosis of subdural haemorrhage. Arch Dis Child 92:468

23. Sperb B (2006) Schädel-Hirn-Trauma bei Kindern. Retrospektive Auswertung der DIVI-Notarztprotokolle aus Bayern. Dissertation, Universität Würzburg

24. Teasdale G, Jennett B (1974) Assessment of coma and impaired consciousness. Lancet 2:81-84

25. Walker PA, Harting MT, Baumgartner JE et al (2009) Modern approaches to pediatric brain injury therapy. JTrauma 67:120-127 